\title{
CARLEMAN AND SEMI-CARLEMAN OPERATORS
}

\section{SCHREIBER AND GY. TARGONSKI ${ }^{1}$}

$1^{\circ}$ Introduction. By a Carleman operator we mean an integral operator $K$ on $L^{2}(a, b),-\infty \leqq a<b \leqq+\infty$, with measurable kernel $k$ such that

$$
\int_{a}^{b}|k(x, y)|^{2} d v<\infty, \text { a.e. } x
$$

If instead of (1) we have

$$
\int_{a}^{b}|k(x, y)|^{2} d x<\infty, \text { a.e. } y
$$

we say $K$ is semi-Carleman. If both (1) and (2) hold we say $K$ is bi-Carleman. The theory of Carleman operators was initiated by T. Carleman in 1923 [1], and extended by J. von Neumann [2], using results of Weyl [3]. In the earlier literature it was always assumed that $k(x, y)=\overline{k(y, x)}$, and it is shown in Stone [4, p. 398] that with this assumption $k$ defines (on a certain domain) a symmetric operator. In the present note we do not include the symmetry condition in our definitions, as indicated above. Carleman operators have been studied in [6], [7], [8], [9], and semi-Carleman operators in [5].

In this note we present several remarks on Carleman, semiCarleman, and bi-Carleman operators. In $2^{\circ}$, we give a characterization, without reference to the kernel, of Carleman operators, with a related result concerning compactness. In $3^{\circ}$, we discuss the question of whether the adjoint of an operator $K$ with kernel $k(x, y)$ is the integral operator with the transposed kernel $k^{*}(x, y)=\overline{k(y, x)}$. In $4^{\circ}$ the invariance with regard to the interval employed of the representation of an operator by a semi-Carleman operator is remarked, extending previous results. In $5^{\circ}$, we correct an error in the paper [5].

$2^{\circ}$ A classic result of von Neumann [2], as extended in [6], states that a normal operator is unitarily equivalent to a Carleman operator if and only if 0 is a limit point in the sense of Weyl [3] of its spectrum. However, this does not characterize Carleman operators. Apart from directly exhibiting a kernel, there is the following criterion, due to

Received by the editors November 4, 1968. 8757.

1 Work supported in part by the National Science Foundation under grant GP 
Korotkov [11]: an operator $K$ on $L^{2}(a, b)$ is a Carleman operator if and only if $|K f(x)| \leqq g(x)$ a.e. for all $f$ in the domain of $K,\|f\| \leqq 1$, where $g$ is measurable and a.e. finite. Another criterion, analogous to the Hilbert-Schmidt property $\left(\sum\left\|K \phi_{n}\right\|^{2}<\infty\right.$ for $\left\{\phi_{n}\right\}$ complete ortho-normal) is the following.

Theorem 2.1 (a). A bounded operator $K$ on $L^{2}(a, b)$ is Carleman if and only if

$$
\sum\left|K \phi_{n}(x)\right|^{2}<\infty \quad \text { a.e. } x
$$

for some complete ortho-normal system $\left\{\phi_{n}\right\}$.

Proof. If $K$ has a Carleman kernel $k$, then

$$
k(x, \cdot)=\sum\left(k(x, \cdot), \Phi_{n}\right) \Phi_{n}, \quad \sum\left|\left(k(x, \cdot), \Phi_{n}\right)\right|^{2}<\infty, \text { a.e. } x,
$$

and $\left(k(x, \cdot), \Phi_{n}\right)=\int k(x, y) \phi_{n}(y) d y=K \phi_{n}(x)$, whence (3).

If (3) holds, define $l_{\phi}(x, y)=\sum K \phi_{n}(x) \overline{\phi_{n}(y)}$. Then $l_{\phi}$ is a Carleman kernel, and $\int l_{\phi}(x, y) \phi_{n}(y) d y=K \phi_{n}(x)$ for all $n$. The theorem now can be derived from the following.

Lemma. If a Carleman operator $A$ agrees with a bounded operator $B$ on a dense set $D$ then $A=B$.

Proof. Let $a(x, y)$ be the kernel of $A$. For any $f \in L^{2}$,

$$
|A f(x)| \leqq\left(\int|a(x, y)|^{2} d y\right)^{1 / 2}\|f\|<\infty \quad \text { a.e. }
$$

and if $f_{n} \rightarrow f$ then similarly $A f_{n}(x) \rightarrow A f(x)$ a.e. Now let $f \in L^{2}$ be arbitrary, $f_{n} \rightarrow f, f_{n} \in D$. Then $A f_{n} \rightarrow A f$ a.e., and $B f_{n} \rightarrow B f$ in norm. But then a subsequence $B f_{n_{k}} \rightarrow B f$ a.e., whence $A f=B f$. Q.E.D.

Returning to the proof of Theorem 2.1 (a), we have the Carleman operator with kernel $l_{\phi}$ agreeing with the bounded operator $K$ on the span of the system $\left\{\phi_{n}\right\}$. Hence by the lemma, the two are equal, and the proof is complete.

REMARK. If (3) holds for one system $\left\{\phi_{n}\right\}$ it holds for all. For the unbounded case we have

Theorem 2.1 (b). Let the operator $K$ on $L^{2}(a, b)$ have dense domain $D_{K} . K$ coincides with a Carleman operator $L$ on a dense set $D \subset D_{K} \cap D_{L}$ if and only if (3) holds for some complete orthonormal set $\left\{\phi_{n}\right\} \subset D_{K}$.

Proof. Since $D_{K}$ is dense, we can choose a countable dense set in $D_{K}$, and apply the Gram-Schmidt process to arrive at an orthonormal set in $D_{K}$. By density it will be complete. 
If $K$ coincides with a Carleman operator $L$ on a dense set $D \subset D_{K}$ $\cap D_{L}$, choose a system $\left\{\phi_{n}\right\} \subset D$ and find (as in Theorem 2.1 (a)) $\sum\left|K \phi_{n}(x)\right|^{2}<\infty$ a.e. $x$.

If (3) holds, define $l_{\phi}$ as before, and observe that the integral operator with kernel $l_{\phi}$ coincides with $K$ on the linear span of the system $\left\{\phi_{n}\right\}$.

A related result is

Theorem 2.2. Suppose $A$ is a normal Carleman operator on $L^{2}(a, b)$, $-\infty<a<b<\infty$, having a complete ortho-normal set of eigenvectors $\left\{\phi_{n}\right\}$. If there exists a function $\psi \in L^{2}$ such that either

(i) $\left|\phi_{n}(x)\right| \leqq|\psi(x)|$ for all $n$ and a.e. $x$, or

(ii) $\left|A \phi_{n}(x)\right| \leqq|\psi(x)|$ for all $n$ and a.e. $x$, then $A$ is compact.

Proof. We use the fact that a normal operator whose spectrum has no limit point but 0 is compact. Let $A \phi_{n}=\lambda_{n} \phi_{n}$. By Theorem 2.1, $\lim \left|A \phi_{n}(x)\right|=\lim \left|\lambda_{n}\right|\left|\phi_{n}(x)\right|=0$, a.e. $x$.

Case (i). If $A$ is not compact, there exists a subsequence $\lambda_{n_{k}}$ with $\left|\lambda_{n_{k}}\right| \geqq \delta>0$. Hence $\left|\phi_{n_{k}}(x)\right| \rightarrow 0$, so by dominated convergence $\left\|\phi_{n_{k}}\right\| \rightarrow 0$, but $\left\|\phi_{n_{k}}\right\|=1$.

Case (ii). Here $\left\|A \phi_{n}\right\|^{2}=\left|\lambda_{n}\right|^{2}\left\|\phi_{n}\right\|^{2} \rightarrow 0$ by dominated convergence, and $\left\|\phi_{n}\right\|=1$, so $\lambda_{n} \rightarrow 0$.

$3^{\circ}$ When is the adjoint $K^{*}$ of an integral operator $K$ with kernel $k$ the integral operator with the kernel $\left.k^{*}(x, y)=\overline{k(y, x}\right)$ ? Two sufficient conditions are

$$
\iint d x d y|k(x, y)|^{2}<\infty
$$

and

$$
\int|k(x, y) \| f(y)| d y \in L^{2}(a, b) \quad \text { for all } f \in L^{2}(a, b) .
$$

The second is Zaanen's property $P[10$, p. 227]. In both cases the result follows by applying Fubini's theorem to the double integral $(K f, g)$. Several weaker statements are possible.

Theorem 3.1. If $K$ is semi-Carleman with finite rank kernel $k$ then $K^{*}$ has kernel $k^{*}$.

Proof. By assumption

$$
k(x, y)=\sum_{1}^{n} \phi_{j}(x) \overline{\psi_{j}(y)}
$$


where the $\phi_{j}$ are ortho-normal and the $\psi_{j}$ need not be $L^{2}$ but $\sum\left|\psi_{j}(y)\right|^{2}$ $<\infty$, a.e. $y$. For $f$ in the domain $D_{K}$ of $K$ (necessarily dense; see [5]) we have $(K f, g)=\left(\sum\left(f, \psi_{j}\right) \phi_{j}, g\right)$. Since $f \in D_{K}$ all $\left|\left(f, \psi_{j}\right)\right|<\infty$. Therefore $\left(f, \sum\left(\phi_{j}, g\right) \psi_{j}\right)$ is finite and equal to $\sum\left(g, \psi_{j}\right)\left(\phi_{j}, g\right)=(K f, g)$. Since $D_{K^{*}}=\left\{g \mid \exists h \forall f \in D_{K} \quad(K f, g)=(f, h)\right\}$, we have $h=K^{*} g$ $=\sum\left(\phi_{j}, g\right) \psi_{j}$, so that $g \in D_{K^{*}}$ if and only if $\sum\left(\phi_{j}, g\right) \psi_{j} \in L^{2}$. Thus $D_{K} \cdot \neq\{0\}$, for it contains in particular all $g$ which are orthogonal to the $\phi_{j}$. For $g \in D_{K^{*}}$ we have $K^{*} g(x)=\int k^{*}(x, y) g(y) d y$, as was to be shown.

THEOREM 3.2. If the adjoint of a bounded Carleman operator is Carleman then the kernel of the adjoint is the transposed conjugate kernel, and thus the operator is bi-Carleman.

Proof. Let $K$ be a bounded Carleman operator with kernel $k$, and suppose $K^{*}$ has a Carleman kernel $l$. Then $\operatorname{Re}(K)=\frac{1}{2}\left(K+K^{*}\right)$ has kernel $\frac{1}{2}\{k(x, y)+l(x, y)\}$ and is selfadjoint. By a recent result of J. Weidmann [12], the kernel of a bounded self adjoint Carleman operator is symmetric. Hence $(1 / 2)\{k+l\}$ and $(1 / 2 i)\{k+l\}$ are both symmetric, whence $k^{*}+l^{*}=k+l$ and $-k^{*}+l^{*}=k-l$. Adding, we have $k^{*}=l$. Q.E.D.

It follows from this that a bounded normal Carleman operator is bi-Carleman, since it is known [6] that the adjoint of a normal Carleman operator is Carleman.

THEOREM 3.3. The adjoint of a bounded bi-Carleman operator is represented by the transposed kernel, and is therefore again a bi-Carleman operator.

Proof. Let $K$ be bounded and bi-Carleman, with kernel $k$. Let $k^{t}(x, y)=\overline{k(y, x)}$ and $K^{t}$ the operator with kernel $k^{t}$. Now $K^{t}$ is semiCarleman in particular, and so $\left\{f \mid K^{t} f \in L^{2}\right\}$ is dense (see [5]). Then $\frac{1}{2}\left(K+K^{t}\right)$ is densely defined and has symmetric Carleman kernel $\frac{1}{2}\left(k+k^{t}\right)$. It follows that there exists a dense set $D \subset L^{2}$ on which $K+K^{t}$ is defined and symmetric $\left[4\right.$, p. 398], and on which $K-K^{t}$ is antisymmetric. Now $K=\frac{1}{2}\left(K+K^{t}\right)+\frac{1}{2}\left(K-K^{t}\right)$ on $D$, and $K^{*}$ $=\frac{1}{2}\left(K+K^{t}\right)-\frac{1}{2}\left(K-K^{t}\right)$ on $D$, so that $K+K^{*}=K+K^{t}$ on $D$. Hence $K^{*}=K^{t}$ on $D$. Thus we have a bounded operator $\left(K^{*}\right)$ equal to a Carleman operator $\left(K^{t}\right)$ on the dense domain $D$. By the lemma of $2^{\circ}, K^{*}=K^{t}$ on all of $L^{2}$, and the proof is complete.

These results have a stronger form, with different proofs, in a forthcoming paper of Weidmann [12].

An example of a bi-Carleman operator which is not of HilbertSchmidt class is afforded by the operator of convolution on $L^{2}(-\infty, \infty)$ by an $L^{2}$ function. 
That the adjoint of this operator is the integral operator with the transposed conjugate kernel may be seen by using the Fourier transform $F$ as follows. Let $L_{\phi}$ denote convolution by $\phi \in L^{2}$, and let $M_{k}$ denote multiplication by the function $k$. We have $F L_{\phi} F^{-1}=M_{F \phi}$ (from the relation $F\left(f_{1} * f_{2}\right)=\left(F f_{1}\right)\left(F f_{2}\right)$ ). Taking adjoints we have $F\left(L_{\phi}\right)^{*} F^{-1}=M_{\overline{F_{\phi}}}$. Let $\bar{\phi}(x)=\overline{\phi(-x)}$. Then $F L_{\tilde{\phi}} F^{-1}=M_{F \tilde{\phi}}$. If we show $M_{F \tilde{\phi}}=M_{\overline{P_{\phi}}}$ then we will have shown that $\left(L_{\phi}\right)^{*}=L_{\tilde{\phi}}$. To show $M_{F \tilde{\phi}}=M_{\overline{F \phi}}$ it suffices to show $\overline{F \phi}=F \tilde{\phi}$. But this is easily seen by direct verification. Now

$$
(L f f)(x)=\int \overline{\phi(y-x) f}(y) d y,
$$

as was to be shown.

$4^{\circ}$ Invariance. In [6] it was shown that certain unitary transformations introduced by von Neumann in [2] preserve the Carleman property (1) of an integral operator. In this section we observe that the same is true for the semi-Carleman property (2). The proof is straightforward, and follows the steps of [6, Appendix 2]. We shall here sketch the main points.

The transformation

$$
\left(U_{a, b} f\right)(x)=(b-a)^{1 / 2} f\left(\frac{x-a}{b-a}\right)
$$

maps $L^{2}(0,1)$ onto $L^{2}(a, b)$ unitarily, and clearly preserves (2). That is, a kernel $k(x, y)$ on $L^{2}(0,1)$ becomes essentially

$$
k\left(\frac{u-a}{b-a}, \frac{v-a}{b-a}\right)
$$

on $L^{2}(a, b)$, and so integrability properties are preserved.

The transformation

$$
\left(N_{1} f\right)(x)=\frac{1}{\sqrt{x}} f(\log x)
$$

maps $L^{2}(-\infty, \infty)$ onto $L^{2}(0, \infty)$ unitarily, and

$$
\left(N_{2} f\right)(x)=\frac{1}{1-x} f\left(\frac{x}{1-x}\right)
$$

maps $L^{2}(0, \infty)$ onto $L^{2}(0,1)$ unitarily. Now a semi-Carleman kernel $k(x, y)$ on $L^{2}(-\infty, \infty)$ becomes $1 /(u v)^{1 / 2} k(\log u, \log v)$ on $L^{2}(0, \infty)$, and 


$$
\int_{0}^{\infty} \frac{1}{u v}|k(\log u, \log v)|^{2} d u=\frac{1}{v} \int_{-\infty}^{\infty}|k(w, \log v)|^{2} d w<\infty
$$

for a.e. $v \in[0, \infty]$, whence the new kernel is semi-Carleman on $L^{2}(0, \infty)$. Similarly, $k(x, y)$ given and semi-Carleman on $L^{2}(0, \infty)$ becomes

$$
k\left(\frac{u}{1-u}, \frac{v}{1-v}\right) \cdot \frac{1}{(1-u)(1-v)}
$$

on $L^{2}(0,1)$, and

$$
\begin{aligned}
& \int_{0}^{1}\left|k\left(\frac{u}{1-u}, \frac{v}{1-v}\right) \frac{1}{(1-u)(1-v)}\right|^{2} d u \\
& =\frac{1}{(1-v)^{2}} \int_{0}^{\infty}\left|k\left(w, \frac{1}{1-v}\right)\right|^{2} d w<\infty
\end{aligned}
$$

for a.e. $v \in[0,1] .{ }^{2}$ Composing these transformations, and using the known result for Carleman operators, we have the following statement.

TheORem 4.1. An operator is unitarily equivalent to a Carleman (semi-Carleman), (bi-Carleman) operator on $L^{2}(-\infty, \infty)$ if and only if it is unitarily equivalent to a Carleman (semi-Carleman) (bi-Carleman) operator on $L^{2}(a, b)$, where $(a, b)$ is any finite or semifinite interval.

$5^{\circ}$ Semi-Carleman representation. In [5] it was falsely claimed that the adjoint of a semi-Carleman operator is densely defined. The error was pointed out by $\mathrm{J}$. Weidmann (private communication). His remark is the following. Let $k(x, y)=\alpha(x) \overline{\beta(y)}$, with $\alpha \in L^{2}$ and $\beta$ locally square integrable but not square integrable, and let $K$ be the operator with kernel $k$. By the proof of Theorem 3.1 specialized to the rank 1 case, we have $D_{K^{*}}=\{g \mid(g, \alpha)=0\}$, which is not a dense set. Because of this, it remains an open question whether semiCarleman operators have closed extensions. Therefore the final statement of $[5, \S 4]$, should read: A closed densely defined operator is representable on $L^{2}(-\infty, \infty)$ as a semi-Carleman operator if and only if it has 0 as a limit point of its spectrum.

The authors wish to thank J. Weidmann for permission to quote his results prior to publication.

: Corresponding statements for unitary mappings from $[-\infty, a]$ and $[a, \infty]$ to $[0, \infty]$ are easily proved: 


\section{REFERENCES}

1. T. Carleman, Sur les équations singulières a noyaux réelles et symétriques, Almqvist and Wiksells, Uppsala, 1923.

2. J.v. Neumann, "Charakterisierung des Spektrums eines Integraloperators," in Collected works. Vol. 4: Continuous geometry and other topics, Pergamon Press, Oxford, 1962, pp. 38-55. MR 28 \#1103.

3. H. Weyl, Rend. Circ. Mat. Palermo 27 (1909), 373-392.

4. M. H. Stone, Linear transformations in Hilbert space, Amer. Math. Soc. Colloq. Publ., vol. 15, Amer. Math. Soc., Providence, R. I., 1932.

5. M. Schreiber, Semi-Carleman operators, Acta Sci. Math. (Szeged) 24 (1963), 82-87. MR 27 \#2861.

6. B. Misra, D. Speiser and Gy. Targonski, Integral operators in the theory of scattering, Helv. Phys. Acta 36 (1963), 963-980. MR 29 \#922.

7. Gy. Targonski, On Carleman integral operators, Proc. Amer. Math. Soc. 18 (1967), 450-456. MR 35 \#5982.

8. - Convergence theorems derived from the theory of Carleman integral operators, Compositio Math. 18 (1967), 148-154. MR 36 \#3176.

9. - Seminar on functional operators and equations, Lecture Notes in Math., no. 33, Springer-Verlag, Berlin, 1967. MR $36 \# 744$.

10. A. C. Zaanen, Linear analysis. Measure and integral, Banach and Hilbert space, linear integral equations, Interscience, New York, North-Holland, Amsterdam and Noordhoff, Groningen, 1953. MR 15, 878.

11. V. B. Korotkov, On integral operators with Carleman kernels, Dokl. Akad. Nauk SSSR 165 (1965), 748-751 =Soviet Math. Dokl. 6 (1965), 1496-1499. MR 35 \#816.

12. J. Weidmann (to appear).

ROCKEFELLER UNIVERSITY AND

FordHAM UNIVERSITY 